\title{
Models for Quadratic Algebras Associated with Second Order Superintegrable Systems in 2D*
}

\author{
Ernest G. KALNINS ${ }^{\dagger}$, Willard MILLER Jr. ${ }^{\ddagger}$ and Sarah POST ${ }^{\ddagger}$ \\ $\dagger$ Department of Mathematics, University of Waikato, Hamilton, New Zealand \\ E-mail: math0236@math.waikato.ac.nz \\ URL: http://www.math.waikato.ac.nz \\ $\ddagger$ School of Mathematics, University of Minnesota, Minneapolis, Minnesota, 55455, USA \\ E-mail: miller@ima.umn.edu,postx052@math.umn.edu \\ URL: http://www.ima.umn.edu/ $\sim$ miller/
}

Received October 25, 2007, in final form January 15, 2008; Published online January 18, 2008

Original article is available at http://www.emis.de/journals/SIGMA/2008/008/

\begin{abstract}
There are 13 equivalence classes of 2D second order quantum and classical superintegrable systems with nontrivial potential, each associated with a quadratic algebra of hidden symmetries. We study the finite and infinite irreducible representations of the quantum quadratic algebras though the construction of models in which the symmetries act on spaces of functions of a single complex variable via either differential operators or difference operators. In another paper we have already carried out parts of this analysis for the generic nondegenerate superintegrable system on the complex 2-sphere. Here we carry it out for a degenerate superintegrable system on the 2 -sphere. We point out the connection between our results and a position dependent mass Hamiltonian studied by Quesne. We also show how to derive simple models of the classical quadratic algebras for superintegrable systems and then obtain the quantum models from the classical models, even though the classical and quantum quadratic algebras are distinct.
\end{abstract}

Key words: superintegrability; quadratic algebras; Wilson polynomials

2000 Mathematics Subject Classification: 20C99; 20C35; 22E70

\section{Introduction}

A classical (or quantum) $m$ th order superintegrable system is an integrable $n$-dimensional Hamiltonian system with potential that admits $2 n-1$ functionally independent constants of the motion, the maximum possible, and such that the constants of the motion are polynomial of at most order $m$ in the momenta. Such systems are of special significance in mathematical physics because the trajectories of the classical motions can be determined by algebraic means alone, whereas the quantum eigenvalues for the energy and the other symmetry operators can also be determined by algebraic methods. In contrast to merely integrable systems, they can be solved in multiple ways. The best known (and historically most important) examples are the classical Kepler system and the quantum Coulomb (hydrogen atom) system, as well as the isotropic oscillator. For these examples $m=2$ and the most complete classification and structure results are known for the second order case. There is an extensive literature on the subject $[1,2,3,4,5,6,7,8,9,10,11,12,13,14,15,16,17,18,19,20,21,22]$, with a recent new burst of activity [23, 24, 25, 26, 27, 28, 29, 30, 31, 32, 33, 34, 35, 36, 37, 38, 39, 40, 41, 42, 43, 44]. All such systems have been classified for real and complex Riemannian spaces with $n=2$ and

\footnotetext{
${ }^{\star}$ This paper is a contribution to the Proceedings of the Seventh International Conference "Symmetry in Nonlinear Mathematical Physics" (June 24-30, 2007, Kyiv, Ukraine). The full collection is available at http://www.emis.de/journals/SIGMA/symmetry2007.html
} 
their associated quadratic algebras of symmetries computed [15, 29, 30, 24, 31]. For nonconstant potentials there are 13 equivalence classes of such stems (under the Stäckel transform between manifolds), 7 with nondegenerate (3-parameter) potentials and 6 with degenerate (1-parameter) potentials $[44,30]$. The constants of the motion for each system generate a quadratic algebra that closes at order 6 in the nondegenerate case and at order 4 in the degenerate case.

The representation theory of such algebras is of great interest because it is this quadratic algebra "hidden symmetry" that accounts for the degeneracies of the energy levels of the quantum systems and the ability to compute all associated spectra of such systems by algebraic means alone. In principle, all of these quadratic algebras can be obtained from the quadratic algebra of a single generic 3-parameter potential on the complex two-sphere by prescribed limit operations and through Stäckel transforms. However, these limiting operations are not yet sufficiently understood. Each equivalence class has special properties, and each of the 13 cases is worthy of study in its own right. A powerful technique for carrying out this study is the use of "one variable models". In the quantum case these are realizations of the quadratic algebra (on an energy eigenspace) in terms of differential or difference operators acting on a space of functions of a single complex variable, and for which the energy eigenvalue is constant. Each model is adapted to the spectral decomposition of one of the symmetry operators, in particular, one that is associated with variable separation in the original quantum system. The possible irreducible representations can be constructed on these spaces with function space inner or bilinear products (as appropriate) and intertwining operators to map the representation space to the solution space of the associated quantum system. (There have been several elegant treatments of the representation theory of some quadratic algebras, e.g. [16, 17, 18, 5, 6, 19, 37]. However these have almost always been restricted to finite dimensional and unitary representations and the question of determining all one variable models has not been addressed.) In the classical case these are realizations of the quadratic algebra (restricted to a constant energy surface) by functions of a single pair of canonical conjugate variables.

In [39] we have already carried out parts of this analysis for the generic nondegenerate superintegrable system on the complex 2 -sphere. There the potential was $V=a_{1} / s_{1}^{2}+a_{2} / s_{2}^{2}+a_{3} / s_{3}^{2}$ where $s_{1}^{2}+s_{2}^{2}+s_{3}^{2}=1$, and the one variable quantum model was expressed in terms of difference operators. It gave exactly the algebra that describes the Wilson and Racah polynomials in their full generality. In this paper we treat a superintegrable case with a degenerate potential. Our example is again on the complex 2-sphere, but now the potential is $V=\alpha / s_{3}^{2}$. Though this potential is a restriction of the generic potential, the degenerate case admits a Killing vector so the quadratic algebra structure changes dramatically. The associated quadratic algebra closes at level 4 and has a richer representation theory than the nondegenerate case. Now we find one variable models for an irreducible representation expressed as either difference or differential operators, or sometimes both. We show that this system can occur in unobvious ways, such as in a position dependent mass Hamiltonian recently introduced by Quesne [37].

The second part of the paper concerns models of classical quadratic algebras. Here we inaugurate this study, in particular its relationship to quantum models of superintegrable systems. We first describe how these classical models arise out of standard Hamilton-Jacobi theory. In $[44,27]$ we have shown that for second order superintegrable systems in two dimensions there is a 1-1 relationship between classical quadratic algebras and quantum quadratic algebras, even though these algebras are not isomorphic. In this sense the quantum quadratic algebra, the spectral theory for its irreducible representations and its possible one variable models are already uniquely determined by the classical system. We make this concrete by showing explicitly how the possible classical models of the classical superintegrable system with potential $V=\alpha / s_{3}^{2}$ lead directly to the possible one variable differential or difference operator models for the quantum quadratic algebra. Then we repeat this analysis for the nondegenerate potential $V=a_{1} / s_{1}^{2}+a_{2} / s_{2}^{2}+a_{3} / s_{3}^{2}$ where the quantum model is essentially the Racah algebra $\mathrm{QR}(3)$ 
and its infinite dimensional extension to describe the Wilson polynomials. Our results show that the Wilson polynomial structure is already imbedded in the classical system with potential $V=a_{1} / s_{1}^{2}+a_{2} / s_{2}^{2}+a_{3} / s_{3}^{2}$, even though this potential admits no Lie symmetries. Thus the properties of the Wilson polynomials in their full generality could have been derived directly from classical mechanics!

This work is part of a long term project to study the structure and representation theory for quadratic algebras associated with superintegrable systems in $n$ dimensions $[23,24,25,26$, $27,35,36]$. The analysis for $n=3$ dimensions will be much more challenging, but also a good indication of behavior for general $n$.

\section{The structure equations for S3}

Up to a Stäckel transform, every 2D second order superintegrable system with nonconstant potential is equivalent to one of 13 systems [44]. There is a representative from each equivalence class on either the complex 2-sphere or complex Euclidean space. In several papers, in particular [15], we have classified all of the constant curvature superintegrable systems, and this paper focuses on two systems contained in that list: S9 and S3. The quadratic algebra of the generic nondegenerate system S9 was already treated in [39] and we will return to it again in this paper. First we study the quadratic algebra representation theory for the degenerate potential S3. This one-parameter potential 2-sphere system corresponds to the potential

$$
V=\frac{\alpha}{s_{3}^{2}}
$$

where $s_{1}^{2}+s_{2}^{2}+s_{3}^{2}=1$ is the imbedding of the sphere in Euclidean space. The quantum degenerate superintegrable system is

$$
H=J_{1}^{2}+J_{2}^{2}+J_{3}^{2}+V(x, y)=H_{0}+V,
$$

where $J_{3}=s_{1} \partial_{s_{2}}-s_{2} \partial_{s_{1}}$ and $J_{2}, J_{3}$ are obtained by cyclic permutations of the indices $1,2,3$. The basis symmetries are

$$
L_{1}=J_{1}^{2}+\frac{\alpha s_{2}^{2}}{s_{3}^{2}}, \quad L_{2}=\frac{1}{2}\left(J_{1} J_{2}+J_{2} J_{1}\right)-\frac{\alpha s_{1} s_{2}}{s_{3}^{2}}, \quad X=J_{3}, \quad H=J_{1}^{2}+J_{2}^{2}+J_{3}^{2}+V,
$$

where $J_{3}=s_{2} \partial_{s_{1}}-s_{1} \partial_{s_{2}}$ plus cyclic permutations. They generate a quadratic algebra that closes at order 4 . The quadratic algebra relations are $[H, X]=\left[H, L_{j}\right]=0$ and

$$
\begin{aligned}
& {\left[L_{1}, X\right]=2 L_{2}, \quad\left[L_{2}, X\right]=-X^{2}-2 L_{1}+H-\alpha,} \\
& {\left[L_{1}, L_{2}\right]=-\left(L_{1} X+X L_{1}\right)-\left(\frac{1}{2}+2 \alpha\right) X}
\end{aligned}
$$

The Casimir relation is

$$
\begin{aligned}
\mathcal{C} \equiv & \frac{1}{3}\left(X^{2} L_{1}+X L_{1} X+L_{1} X^{2}\right)+L_{1}^{2}+L_{2}^{2}-H L_{1}+\left(\alpha+\frac{11}{12}\right) X^{2}-\frac{1}{6} H \\
& +\left(\alpha-\frac{2}{3}\right) L_{1}-\frac{5 \alpha}{6}=0 .
\end{aligned}
$$

We know that the quantum Schrödinger equation separates in spherical coordinates, and that corresponding to a fixed energy eigenvalue $H$, the eigenvalues of $X$ take the linear form

$$
\lambda_{n}=\mathcal{A} n+\mathcal{B},
$$


where $n$ is an integer, so we will look for irreducible representations of the quadratic algebra such that the representation space has a basis of eigenvectors $f_{n}$ with corresponding eigenvalues $\lambda_{n}$. (Indeed, from the analysis of [16] or of [6] the structure equations imply that the spectrum of $X$ must be of this form.) We will use the abstract structure equations to list the corresponding representations and compute the action of $L_{1}$ and $L_{2}$ on an $X$ basis. Thus, we assume that there is a basis $\left\{f_{n}\right\}$, for the representation space such that

$$
X f_{n}=\lambda_{n} f_{n}, \quad L_{1} f_{n}=\sum_{j} C(j, n) f_{j}, \quad L_{2} f_{n}=\sum_{j} D(j, n) f_{j} .
$$

Here, $\mathcal{A}, \mathcal{B}$ are not yet fixed. We do not impose any inner product space structure.

From these assumptions we can compute the action of $L_{1}$ and $L_{2}$ on the basis. Indeed,

$$
\begin{aligned}
& {\left[L_{1}, L_{2}\right] f_{n}=\sum_{j, k}(C(j, k) D(k, n)-D(j, k) C(k, n)) f_{j},} \\
& {\left[L_{1}, X\right] f_{n}=\sum_{j}\left(\lambda_{n}-\lambda_{j}\right) C(j, n) f_{j}, \quad\left[L_{2}, X\right] f_{n}=\sum_{j}\left(\lambda_{n}-\lambda_{j}\right) D(j, n) f_{j} .}
\end{aligned}
$$

On the other hand, from the equations (1) we have

$$
\begin{aligned}
& {\left[L_{1}, X\right] f_{n}=2 \sum_{j} D(j, n) f_{j},} \\
& {\left[L_{2}, X\right] f_{n}=-2 \sum_{j} C(j, n) f_{j}+\left(-\lambda_{n}^{2}+H-\alpha\right) f_{n},} \\
& {\left[L_{1}, L_{2}\right] f_{n}=-\sum_{j}\left(\lambda_{n}+\lambda_{j}\right) C(j, n) f_{j}-\left(\frac{1}{2}+2 \alpha\right) \lambda_{n} f_{n} .}
\end{aligned}
$$

Now we equate equations (4) with (5) or (6). For $j=n$, equating coefficients of $f_{n}$ in the resulting identities yields the conditions

$$
D(n, n)=0, \quad C(n, n)=\frac{-\lambda_{n}^{2}+H-\alpha}{2} .
$$

Similarly, equating coefficients of $f_{j}$ in the case $j \neq n$ yields

$$
\mathcal{A}(n-j) D(j, n)=-2 C(j, n), \quad \mathcal{A}(n-j) C(j, n)=2 D(j, n),
$$

or

$$
\left(\mathcal{A}^{2}(n-j)^{2}+4\right) C(j, n)=0, \quad j \neq n .
$$

Thus, either $C\left(j, n\right.$ and $D(j, n)$ vanish or $\mathcal{A}^{2}(n-j)^{2}=-4$. We can scale $\mathcal{A}$ such that the smallest nonzero jump is for $j=n \pm 1$, in which case $\mathcal{A}= \pm 2 i$. By replacing $n$ by $-n$ if necessary, we can assume $\mathcal{A}=2 i$. (We also set $\mathcal{B}=i \mu$.) Thus the only possible nonzero values of $C(j, n)$, $D(j, n)$ are for $j=n, n \pm 1$ and there are the relations

$$
D(n+1, n)=-i C(n+1, n), \quad D(n-1, n)=i C(n-1, n) .
$$

Comparing (3) and (7) and equating coefficients of $f_{n \pm 2}, f_{n \pm 1}$, respectively, on both sides of the resulting identities, we do not obtain new conditions. However, equating coefficients of $f_{n}$ results in the condition

$$
F_{n+1}-F_{n}=\frac{1}{2}(2 n+\mu)\left(4 n^{2}+4 \mu n+\mu^{2}+H+\alpha+\frac{1}{2}\right),
$$


where $F_{n}=C(n, n-1) C(n-1, n)$. The general solution of this difference equation is

$$
\begin{aligned}
F_{n}= & n^{4}+(2 \mu-2) n^{3}+\left(\frac{3}{2} \mu^{2}-3 \mu+\frac{H}{2}+\frac{\alpha}{2}+\frac{5}{3}\right) n^{2} \\
& +\left(\frac{\mu^{3}}{2}-\frac{3 \mu^{2}}{2}+\left[\frac{H}{2}+\frac{\alpha}{2}+\frac{5}{4}\right] \mu-\frac{H}{2}-\frac{\alpha}{2}-\frac{1}{4}\right) n+\kappa,
\end{aligned}
$$

where $\kappa$ is an arbitrary constant.

To determine $\kappa$ we substitute these results into the Casimir equation (2) and set equal to zero the coefficients of $f_{j}$ in the expression $\mathcal{C} f_{n}=0$. For $j \neq n$ we get nothing new. However, $j=n$ we find

$$
\begin{aligned}
\kappa= & \frac{1}{16} \mu^{4}-\frac{1}{4} \mu^{3}+\frac{H+\alpha+5 / 2}{8} \mu^{2}-\frac{1 / 2+H+\alpha}{4} \mu \\
& +\frac{1}{8} H+\frac{\alpha}{8}+\frac{H^{2}}{16}-\frac{H \alpha}{8}+\frac{\alpha^{2}}{16} .
\end{aligned}
$$

Thus, $F_{n}=C(n, n-1) C(n-1, n)$ is an explicit 4 th order polynomial in $n$. By factoring this polynomial in various ways, and re-normalizing the basis vectors $f_{n}$ appropriately via $f_{n} \rightarrow$ $c(n) f_{n}$, we can achieve a realization of the action of $L_{1}$ and $L_{2}$ such that

$$
\begin{aligned}
& L_{1} f_{n}=C(n+1, n) f_{n+1}+C(n, n) f_{n}+C(n-1, n) f_{n-1}, \\
& L_{2} f_{n}=D(n+1, n) f_{n+1}+D(n, n) f_{n}+D(n-1, n) f_{n-1}
\end{aligned}
$$

and all of the coefficients are polynomials in $n$. The 4 roots of $F_{n}$ are

$$
\frac{1-\mu}{2} \pm \frac{1}{4} \sqrt{2-4(H+\alpha) \pm 2 \sqrt{1-4(H+\alpha)+16 H \alpha}}
$$

so a convenient factorization is

$$
\begin{aligned}
& C(n, n-1)=\left(n+\frac{\mu-1}{2}\right)^{2}-\frac{1}{8}(1-2(H+\alpha)+\sqrt{1-4(H+\alpha)+16 H \alpha}), \\
& C(n-1, n)=\left(n+\frac{\mu-1}{2}\right)^{2}-\frac{1}{8}(1-2(H+\alpha)-\sqrt{1-4(H+\alpha)+16 H \alpha}) .
\end{aligned}
$$

From these expressions and from

$$
D(n, n)=0, \quad C(n, n)=\frac{(2 n+\mu)^{2}+H-\alpha}{2}, \quad D(n \pm 1, n)=\mp i C(n \pm 1, n)
$$

we see that we can find $C, D$ coefficients in which the the dependence on $n$ is always as a polynomial.

There are raising and lowering operators

$$
A^{\dagger}=L_{1}+i L_{2}+\frac{1}{2}\left(X^{2}-H+\alpha\right), \quad A=L_{1}-i L_{2}+\frac{1}{2}\left(X^{2}-H+\alpha\right) .
$$

Indeed,

$$
A^{\dagger} f_{n}=2 C(n+1, n) f_{n+1}, \quad A f_{n}=2 C(n-1, n) f_{n-1},
$$

and $\left[A, A^{\dagger}\right] f_{n}=2\left(F_{n+1}-F_{n}\right) f_{n}$, so $\left[A, A^{\dagger}\right]$ is a third order polynomial in $X$. 
To get a one-variable model of the quadratic algebra in terms of second order differential operators, we can simply make the choices $f_{n}(t)=t^{n}, X=i\left(2 t \frac{d}{d t}+\mu\right)$ and define $L_{1}$ from expressions (9) via the prescription

$$
L_{1} f_{n}(t)=\left(t C\left(t \frac{d}{d t}+1, t \frac{d}{d t}\right)+C\left(t \frac{d}{d t}, t \frac{d}{d t}\right)+t^{-1} C\left(t \frac{d}{d t}-1, t \frac{d}{d t}\right)\right) f_{n}(t)
$$

with a similar procedure for $L_{2}$.

In general the irreducible representations that we have defined are infinite dimensional and the basis vectors $f_{n}$ occur for all positive and negative integers $n$. We can obtain representations bounded below, and with lowest weight $\mu$ for $-i X$ and corresponding lowest weight vector $f_{0}$, simply by requiring $F_{0}=0$, which amounts to setting $\kappa=0$. For convenience we set $\alpha=1 / 4-a^{2}$. Then we have

$$
F_{n}=C(n, n-1) C(n-1, n)=n(n+\mu-1)(n+\mu-1+a)(n-a) .
$$

Since $\kappa=0,(8), H$ must be a solution of this quadratic equation:

$$
H=-(\mu-1+a)^{2}+\frac{1}{4}
$$

A convenient choice is

$$
\begin{aligned}
& C(n-1, n)=n(n+\mu-1+a), \quad C(n+1, n)=(n+\mu)(n+1-a), \\
& C(n, n)=2 n^{2}+2 n \mu-\mu a+a+\mu-\frac{1}{2} .
\end{aligned}
$$

If $\mu$ is not a negative integer then this bounded below representation is infinite dimensional. However, if there is a highest weight vector $f_{m}$ then we must have $F_{m+1}=0$, or $\mu=-m$, $m=0,1, \ldots$. Thus the finite dimensional representations are indexed by the nonnegative integer $m$ and the eigenvalues of $-i X$ are $m, m-2, \ldots,-m$. The dimension of the representation space is $m+1$.

At this point it is worth pointing out that all of the finite dimensional, infinite dimensional bounded below, and general infinite dimensional irreducible representations and models of the quadratic algebra associated with the superintegrable system S3 are of direct interest and applicability. A similar argument was made in [39] where we gave examples of various analytic function expansions and distinct unitary structures associated with one superintegrable system. The original S3 quantum system is given in terms of complex variables with no specific inner product structure imposed. One could use the representation theory results to describe eigenfunction expansions simply in terms of analytic functions. If one wants an inner product structure or bilinear product structure, it is merely necessary to impose the structure on a single eigenspace of $H$, and there are a variety of ways to do this. For example, one could restrict the complex system to the real sphere and impose the standard inner product for that case. Alternatively, one could restrict to the real hyperboloid of one sheet, or the real hyperboloid of two sheets. In all cases the models of the irreducible representations are relevant, though not necessarily to one special case, such as the real sphere with the standard inner product. While we have no direct proof that all models of irreducible representations of quadratic algebras obtained in this way lead to representations for some version of the original superintegrable system, we have no counterexamples. For a deeper analysis we need to construct intertwining operators that relate basis functions for the model with eigenfunctions of the quantum Hamiltonian. 


\section{Differential operator models}

A convenient realization of the finite dimensional representations by differential operators in one complex variable is

$$
\begin{aligned}
L_{1}= & \left(t^{3}+2 t^{2}+t\right) \frac{d^{2}}{d t^{2}}+\left((2-a-m) t^{2}+2(1-m) t+a-m\right) \frac{d}{d t} \\
& +m(a-1) t+a(m+1)-m-\frac{1}{2}, \quad X=i\left(2 t \frac{d}{d t}-m\right), \\
L_{2}= & i\left(-t^{3}+t\right) \frac{d^{2}}{d t^{2}}+i\left((a+m-2) t^{2}+a-m\right) \frac{d}{d t}-i m(a-1) t .
\end{aligned}
$$

This model is also correct for infinite dimensional bounded-below representations, except that now the lowest weight is $\mu=-m$ where $m \neq 0,1,2, \ldots$ is a complex number. The raising and lowering operators for the model are

$$
A^{\dagger}=2 t^{3} \frac{d^{2}}{d t^{2}}+2(2-a-m) t^{2} \frac{d}{d t}+2 m(a-1) t, \quad A=2 t \frac{d^{2}}{d t^{2}}+2(a-m) \frac{d}{d t} .
$$

In the finite dimensional case, for example, the eigenvalues of $L_{1}$ are

$$
\chi_{n}=a^{2}-\frac{1}{4}-\left(n-a+\frac{1}{2}\right)^{2}, \quad n=0,1, \ldots, m,
$$

and the corresponding unnormalized eigenfunctions are

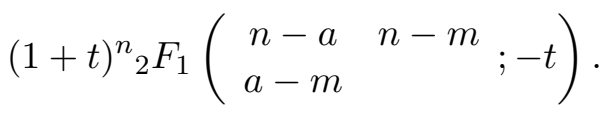

Now, motivated by the quantum mechanical system on the real 2 -sphere, we impose a Hilbert space structure on the irreducible representations such that $L_{1}$ and $L_{2}$ are self-adjoint and $X$ is skew adjoint:

$$
\left\langle L_{j} f_{n}, f_{n^{\prime}}\right\rangle=\left\langle f_{n}, L_{j} f_{n^{\prime}}\right\rangle, \quad j=1,2 ; \quad\left\langle X f_{n}, f_{n^{\prime}}\right\rangle=-\left\langle f_{n}, X f_{n^{\prime}}\right\rangle .
$$

Writing $\phi_{n}=k_{n} f_{n}$ where $\phi_{n}$ has norm 1, we have the recursion relation

$$
k_{n}^{2}=\frac{(n-1+\mu)(n-a)}{n(n-1+\mu+a)} k_{n-1}^{2} .
$$

For infinite dimensional bounded below representations $k_{n}^{2}$ must be positive for all integers $n \geq 0$, and we normalize $k_{0}=1$. Thus

$$
k_{n}^{2}=\frac{(\mu)_{n}(1-a)_{n}}{n !(a+\mu)_{n}} .
$$

For finite dimensional representations we have $\mu=-m$. Normalizing $k_{0}=1$, (possible for $a<1$ or for $a>m$ ), we find that an orthonormal basis in the one variable model is given by $\phi_{n}(t)=k_{n} f_{n}(t)=k_{n} t^{n}, n=0,1, \ldots, m$ where

$$
k_{n}=\sqrt{\frac{(-m)_{n}(1-a)_{n}}{n !(-m+a)_{n}}}=\sqrt{\frac{m !(1-a)_{n}(1-a)_{m-n}}{(1-a)_{m} n !(m-n) !}} .
$$

Note the reflection symmetry $\left\|f_{n}\right\|=\left\|f_{m-n}\right\|$. 
To derive a realization of the Hilbert space for the differential operator models of the finite dimensional and infinite dimensional bounded below unitary representations in terms of a function space inner product

$$
\langle p, q\rangle=K \iint p(t) \overline{q(t)} \rho(t \bar{t}) d t d \bar{t},
$$

where $p, q$ are polynomials and $K$ is a normalization constant, we use the formal self- and skew-adjoint requirements and obtain a differential equation for the weight function:

$$
\left(-\zeta^{2}+\zeta\right) \frac{d^{2} \rho(\zeta)}{d \zeta^{2}}+(-\mu-a+1+(-1+\mu-a) \zeta) \frac{d \rho(\zeta)}{d \zeta}+(-2+\mu-2 a+a \mu) \rho(\zeta)=0,
$$

where $\zeta=t \bar{t}$. The solution that vanishes at $\zeta=1$ for $a<1 / 2$ and is integrable at $\zeta=0$ for $a+\mu>-1$ is

$$
\rho_{1}(\zeta)=(1-\zeta)^{1-2 a}{ }_{2} F_{1}\left(\begin{array}{ll}
-\frac{\mu+3 a-Q}{2}+1, & -\frac{\mu+3 a+Q}{2}+1 \\
2-2 a & ; 1-\zeta
\end{array}\right),
$$

where $Q=\sqrt{a^{2}+(2 \mu-8) a+\mu^{2}+4 \mu-8}$. (Note that the integral is an even function of $Q$.) At $\zeta=0$ this function has a branch point with behavior $\zeta^{a+\mu}$. We write $t=r e^{i \theta}, \bar{t}=r e^{-i \theta}$, $\zeta=r^{2}$ and choose our contours of integration for the inner product as the unit circle $\left|e^{i \theta}\right|=1$, i.e., $0 \leq \theta \leq 2 \pi$ and, in the complex $\zeta$-plane, a contour that starts at $\zeta=1$ and travels just above the real $\zeta$-axis to circle $\zeta=0$ once in the counterclockwise direction and returns to $\zeta=1$ just below the real $\zeta$-axis. We require that $\langle 1,1\rangle=1$. By choosing a regime where $a+\mu>-1$ we can shrink the $\zeta$-contour about $\zeta=0$ so that the norm takes the form

$$
\begin{aligned}
\langle 1,1\rangle & =-4 \pi K_{1} e^{i \pi(a+\mu)} \sin [\pi(a+\mu)] \int_{0}^{1} \rho_{1}(\zeta) d \zeta \\
& =\frac{-\pi K_{1}}{a-1} e^{i \pi(a+\mu)} \sin [\pi(a+\mu)]_{2} F_{1}\left(\frac{-\mu-3 a+Q}{2}+1 \frac{-\mu-3 a-Q}{2}+1 ; 1\right) \\
& =4 \pi K_{1} e^{i \pi(a+\mu)} \sin [\pi(a+\mu)] \frac{\Gamma(2-2 a) \Gamma(a+\mu+1)}{\Gamma\left(2-\frac{a-\mu+Q}{2}\right) \Gamma\left(2-\frac{a-\mu-Q}{2}\right)},
\end{aligned}
$$

where we have integrated term-by-term and then made use of Gauss' Theorem for the summation of ${ }_{2} F_{1}(1)$. This gives us the value for $K_{1}$ such that $\langle 1,1\rangle=1$. Now, the result extends for the original contour by analytic continuation. This defines a pre-Hilbert space inner product that then can be extended to obtain a true Hilbert space.

The contour integral for the inner product obtained in the previous paragraph requires Re $a<1$ for convergence, and this doesn't hold for some of the unitary irreducible representations defined above. Accordingly, we consider a second solution of the weight function equation. The solution that vanishes at $\zeta=0$ for $a+\mu>0$ and is integrable at $\zeta=1$ for $a<1$ is

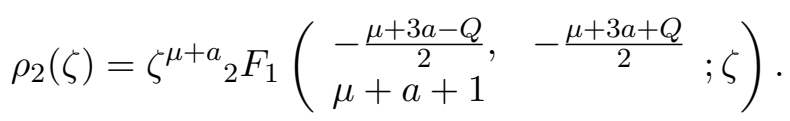

At $\zeta=1$ this function has a branch point with behavior $(1-\zeta)^{1-2 a}$. We write $t=r e^{i \theta}, \bar{t}=r e^{-i \theta}$, $\zeta=r^{2}$ and choose our contours of integration for the inner product as the unit circle $\left|e^{i \theta}\right|=1$ and, in the complex $\zeta$-plane, a contour that starts at $\zeta=0$ and travels just below the real $\zeta$-axis to circle $\zeta=1$ once in the counterclockwise direction and returns to $\zeta=0$ just above the real $\zeta$-axis. This integral converges for $\operatorname{Re}(a+\mu)>-1$. We require that $\langle 1,1\rangle=1$. By choosing a regime where $a<1$ we can shrink the $\zeta$-contour about $\zeta=1$ so that the norm takes the form

$$
\langle 1,1\rangle=4 \pi K_{2} e^{i \pi(2 a-1)} \sin [\pi(2 a-1)] \int_{0}^{1} \rho_{2}(\zeta) d \zeta
$$




$$
\begin{aligned}
& =\frac{\pi K_{2}}{\mu+a+1} e^{i \pi(2 a-1)} \sin [\pi(2 a-1)]_{2} F_{1}\left(\begin{array}{ll}
\frac{\mu+3 a+Q}{2} & \frac{\mu+3 a-Q}{2} \\
a+\mu+2 & 1
\end{array}\right) \\
& =-4 \pi K_{2} e^{i \pi(2 a-1)} \sin [\pi(2 a-1)] \frac{\Gamma(2-2 a) \Gamma(a+\mu+1)}{\Gamma\left(2-\frac{a-\mu+Q}{2}\right) \Gamma\left(2-\frac{a-\mu-Q}{2}\right)} .
\end{aligned}
$$

This gives us the value for $K$ such that $\langle 1,1\rangle=1$, and the result extends by analytic continuation to all values of $a, \mu$ for which the original contour integral converges.

Thus we have an explicit pre-Hilbert function space inner product for each of our differential operator models. In the finite dimensional case we have the reproducing kernel function

$$
\delta(t, \bar{s})=\sum_{n=0}^{m} \phi_{n}(t) \bar{\phi}_{n}(s)={ }_{2} F_{1}\left(\begin{array}{cc}
-m & 1-a \\
-m+a & ; t \bar{s}
\end{array} .\right.
$$

In the infinite dimensional bounded below case we have the reproducing kernel function

$$
\delta(t, \bar{s})=\sum_{n=0}^{\infty} \phi_{n}(t) \bar{\phi}_{n}(s)={ }_{2} F_{1}\left(\begin{array}{cc}
\mu & 1-a \\
\mu+a & ; t \bar{s}
\end{array}\right)
$$

which converges as an analytic function and in the Hilbert space norm for $|s|<1$. Here,

$$
\|\delta(s, \bar{s})\|={ }_{2} F_{1}\left(\begin{array}{cc}
\mu & 1-a \\
\mu+a & ;|s|^{2}
\end{array}\right) .
$$

In each case $\langle f(t), \delta(t, \bar{s})\rangle=f(s)$ for $f$ in the Hilbert space.

\section{Difference operator models}

There are also difference operator models for the representations of the S3 quadratic algebra. We first give the details for the finite dimensional representations indexed by the nonnegative integer $m$. Here the operator $L_{1}$ is diagonalized:

$$
\begin{aligned}
& L_{1}=-\lambda(t)+a-\frac{1}{2}, \quad \lambda(t)=t(t-2 a+1), \\
& -i X=\frac{(t-2 a+1)(t-m)}{2 t-2 a+1} T^{1}-\frac{t(t+m-2 a+1)}{2 t-2 a+1} T^{-1}, \\
& L_{2}=\frac{(t-a+1)(t-2 a+1)(t-m)}{2 t-2 a+1} T^{1}+\frac{t(t-a)(t+m-2 a+1)}{2 t-2 a+1} T^{-1},
\end{aligned}
$$

where $T^{k}$ is the difference operator $T^{k} f(t)=f(t+k)$. The basis functions are $f_{n}(t)=$ $(-1)^{n} p_{n}(\lambda)$ where

$$
p_{n}(\lambda(t))={ }_{3} F_{2}\left(\begin{array}{lll}
-n & -t & t-2 a+1 \\
-m & 1-a &
\end{array}\right) .
$$

Here $f_{n}$ is a polynomial of order $n$ in the variable $\lambda(t)$, a special case of the family of dual Hahn polynomials $[45$, p. 346]. These polynomials are orthogonal with respect to a measure with support at the values $t=0,1, \ldots, m$, in agreement with equation (14) for the eigenvalues of $L_{1}$. Indeed, we have (for $a<1$ )

$$
\sum_{t=0}^{m} \frac{(1-2 a)_{t}(3 / 2-a)_{t}(-m-1)_{t}(-1)^{t}}{(1 / 2-a)_{t}(2+m-2 a)_{t} t !} p_{n}(\lambda(t)) p_{n^{\prime}}(\lambda(t))=\frac{(2-2 a)_{m}(a-m)_{n} n !}{(1-a)_{m}(1-a)_{n}(-m)_{n}} \delta_{n n^{\prime}} .
$$


For the infinite dimensional, bounded below, case we have

$$
\begin{aligned}
L_{1}= & t^{2}+a^{2}-\frac{1}{4}, \\
-i X= & \frac{(1 / 2-a-i t)(\mu+a-1 / 2-i t)}{2 t} T^{i}-\frac{(1 / 2-a+i t)(\mu+a-1 / 2+i t))}{2 t} T^{-i}, \\
L_{2}= & -i \frac{(1-2 i t)(1 / 2-a-i t)(\mu+a-1 / 2-i t)}{4 t} T^{i} \\
& -i \frac{(1+2 i t)(1 / 2-a+i t)(\mu+a-1 / 2+i t))}{4 t} T^{-i} .
\end{aligned}
$$

The basis functions are $f_{n}(t)=(-1)^{n} s_{n}\left(t^{2}\right)$ where

$$
s_{n}\left(t^{2}\right)={ }_{3} F_{2}\left(\begin{array}{lll}
-n & \frac{1}{2}-a+i t & \frac{1}{2}-a-i t \\
\mu & 1-a & ; 1
\end{array}\right) .
$$

Here $f_{n}$ is a polynomial of order $n$ in the variable $t^{2}$, a special case of the family of continuous dual Hahn polynomials [45, p. 331]. The orthogonality and normalization are given by

$$
\begin{aligned}
& \frac{1}{2 \pi} \int_{0}^{\infty}\left|\frac{\Gamma(1 / 2-a+i t) \Gamma(\mu+a-1 / 2+i t) \Gamma(1 / 2+i t)}{\Gamma(2 i t)}\right|^{2} s_{n}\left(t^{2}\right) s_{n^{\prime}}\left(t^{2}\right) d t \\
& \quad=\frac{\Gamma(n+\mu) \Gamma(n+1-a) \Gamma(n+\mu+a) n !}{(\mu)_{n}^{2}\left|(1-a)_{n}\right|^{2}} \delta_{n n^{\prime}},
\end{aligned}
$$

\begin{tabular}{|c|c|c|}
\hline representation & parameter range & model \\
\hline finite dimensional & $\begin{array}{l}\mu=-m, m=0,1,2, \ldots \\
\text { either } a<1 \text { or } a+\mu>0\end{array}$ & $\begin{array}{l}\text { differential operators } \\
\text { difference operators }\end{array}$ \\
\hline inf. dim. bdd. below & $\begin{array}{c}\mu>0 \\
a<1 \text { and } a+\mu>0\end{array}$ & $\begin{array}{c}\text { differential operators } \\
\text { difference operators }\end{array}$ \\
\hline inf, dim. bdd. below & $\begin{array}{c}0>\mu=-n_{0}+t, t \in(0,1) \\
a=n_{0}+s, s \in(0,1)\end{array}$ & differential operators \\
\hline inf. dim. bdd. below & $\begin{array}{c}0>\mu=-n_{0}+t, t \in(0,1) \\
-t<a<1-t\end{array}$ & differential operators \\
\hline
\end{tabular}

where $\mu>1 / 2-a>0$.

In summary, we have found the following possibilities for bounded below irreducible representations such that $L_{1}, L_{2}$ are self-adjoint and $X$ is skew adjoint, together with associated one variable models. (Here, $n_{0}$ is a positive integer.)

\section{Quesne's position dependent mass (PDM) system in a two-dimensional semi-infinite layer}

In [37] Quesne considered a superintegrable exactly solvable position dependent mass (PDM) system in a two-dimensional semi-infinite layer. Her system is equivalent via a gauge transformation to a standard quantum mechanical problem on the real 2-sphere with potential of the form S3. Indeed, in Quesne's paper we are given the Hamiltonian

$$
-H_{Q}=\cosh ^{2} q x\left(\partial_{x}^{2}+\partial_{y}^{2}\right)+2 q \cosh q x \sinh q x \partial_{x}+q^{2} \cosh ^{2} q x-\frac{q^{2} k(k-1)}{\sinh ^{2} q x} .
$$


We adopt coordinates on the unit sphere as

$$
s_{1}=\frac{\sin q y}{\cosh q x}, \quad s_{2}=\frac{\cos q y}{\cosh q x}, \quad s_{3}=\tanh q x,
$$

where $s_{1}^{2}+s_{2}^{2}+s_{3}^{2}=1$ and the metric is $d s^{2}=q^{2}\left(d x^{2}+d y^{2}\right) / \cosh ^{2} q x$. The Laplacian becomes

$$
\Delta_{S}=\frac{\cosh ^{2} q x}{q^{2}}\left(\partial_{x}^{2}+\partial_{y}^{2}\right)
$$

In these coordinates, the degenerate superintegrable system $\mathrm{S} 3$ becomes

$$
H_{S}=\frac{\cosh ^{2} q x}{q^{2}}\left(\partial_{x}^{2}+\partial_{y}^{2}\right)+\frac{\frac{1}{4}-\alpha^{2}}{\tanh ^{2} q x} .
$$

By a gauge transform $H_{O}=(\cosh q x)^{-1} H_{S} \cosh q x$, we get

$$
H_{O}=\frac{1}{q^{2}}\left\{\cosh ^{2} q x\left(\partial_{x}^{2}+\partial_{y}^{2}\right)+2 q \cos q x \sinh q x \partial_{x}+q^{2} \cosh ^{2} q x+q^{2} \frac{\frac{1}{4}-\alpha^{2}}{\sinh ^{2} q x}\right\}+\frac{1}{4}-\alpha^{2} .
$$

Thus we have $H_{Q}=-q^{2} H_{0}+q^{2}\left(1 / 4-\alpha^{2}\right)=-q^{2}(\cosh q x)^{-1} H_{S} \cosh q x+q^{2}\left(1 / 4-\alpha^{2}\right)$, with $1 / 4-\alpha^{2}=-k(k-1)$ which has solutions $k=a+1 / 2$ or $k=-a+1 / 2$. Since $k$ is assumed positive and $a$ is required to be less than 1 , we take $a<0$.

Suppose we find an eigenvector for $H_{S}$ with eigenvalue $\lambda_{S}$, call it $v_{\lambda_{S}}$. Then $\lambda_{Q}$ will be the eigenvalue of $v_{\lambda_{Q}}$ for $H_{Q}$. We have the transformations $v_{\lambda_{Q}}=v_{\lambda S} / \cosh q x$ and $\lambda_{Q}=-q^{2} \lambda_{S}+$ $q^{2}\left(1 / 4-\alpha^{2}\right)=-q^{2} \lambda_{S}-q^{2} k(k-1)$. Checking the two eigenvalues, we have $\lambda_{S}=-(\mu-1+a)^{2}+1 / 4$ and $\lambda_{Q}=q^{2}(N+2)(N+2 k+1)$. We note that these two values coincide when $-\mu=m=N+1$ with $m$ an integer.

Using the above calculations and the eigenfunctions given in the paper, we can obtain eigenfunctions for the S3 case as

$$
v_{\lambda_{S}}=N_{n, \ell}^{(k)}(\tanh q x)^{-a+\frac{1}{2}}(\cosh q x)^{-\ell-1} P_{n}^{-a, \ell+1}\left(-\tanh ^{2} q x\right) \chi_{\ell}(y),
$$

or in coordinates on the sphere

$$
v_{\lambda_{S}}=N_{n, \ell}^{(k)}\left(s_{3}\right)^{-a+\frac{1}{2}}\left(s_{1}^{2}+s_{2}^{2}\right)^{\frac{\ell+1}{2}} P_{n}^{-a, \ell+1}\left(-s_{3}^{2}\right) \chi_{\ell}(y),
$$

where $m=2 n+\ell+1$, and $\chi_{\ell}(y)=\sin [(\ell+1) q y]$ or $\cos [(\ell+1) q y]$. We can rewrite these by noting $1 / \cosh ^{2} q x=1-\tanh ^{2} q x$ so that we can write $\sin q y=s_{1} / \sqrt{1-s_{3}^{2}}$ and $\cos q y=s_{2} / \sqrt{1-s_{3}^{2}}$, then we obtain

$$
\chi_{\ell}(y)=a_{n} T_{\ell+1}\left(\frac{s_{2}}{\sqrt{s_{1}^{2}+s_{2}^{2}}}\right)+b_{n} \frac{s_{1}}{\sqrt{s_{1}^{2}+s_{2}^{2}}} U_{\ell}\left(\frac{s_{2}}{\sqrt{s_{1}^{2}+s_{2}^{2}}}\right),
$$

where $T_{\ell}$ and $U_{\ell}$ are the Chebyshev polynomials of the first and second kind, respectively.

Quesne found the S3 quadratic algebra (which closes at order 4) but did not use it for spectral analysis purposes because her problem involved a boundary condition that broke the full quadratic algebra symmetry. Instead she considered her system as a special case of S9 and used the more complicated S9 symmetry algebra that closes at order 6 to find the finite dimensional representations. (Note that although the 1-parameter S3 potential is a limit of the 3parameter S9 potential as two of the parameters go to 0 , a discontinuity occurs in the structure of the quadratic algebra. A first order symmetry appears and the number of second order symmetries jumps from 3 to 4.) Quesne's point of view has merit, but it complicates the spectral 
analysis of the problem, since the only one-variable model is in terms of difference operators and Racah polynomials. From our vantage point of one one variable differential operator analysis for the model, Quesne's boundary conditions amount to decomposing an irreducible subspace corresponding to an $m$-dimensional representation into a direct sum of even and odd parity subspaces $V^{+}, V^{-}$. (Indeed her boundary conditions require choice of $\chi_{\ell}(y)$ in the cosine form for $\ell$ even and in the sine form for $\ell$ odd.) Let $P$ be the operator $P f(t)=t^{m} f(1 / t)$. Since $P^{2}=I$ and $\left\|f_{n}\right\|=\left\|f_{m-n}\right\|$, it is clear that $P$ is unitary. We define unit vectors $\Phi_{\ell}^{+}=2^{-1 / 2}\left(\phi_{\ell}+(-1)^{m} \phi_{m-\ell}\right)$ and $\Phi_{\ell}^{-}=2^{-1 / 2}\left(\phi_{\ell}-(-1)^{m} \phi_{m-\ell}\right)$ for $\ell=0,1, \ldots,[m / 2]$. Then for $m=2 k$ the vectors $\Phi_{\ell}^{+}, \ell=0, \ldots, k$ form an orthonormal basis for $V_{m}^{+}$and the vectors $\Phi_{\ell}^{-}$, $\ell=0, \ldots, k-1$ form an on basis for $V_{m}^{-}$. For $m=2 k-1$, the vectors $\Phi_{\ell}^{+}, \ell=0, \ldots, k-1$ form an $\mathrm{ON}$ basis for $V_{m}^{+}$and the vectors $\Phi_{\ell}^{-}, \ell=0, \ldots, k-1$ form an orthonormal basis for $V_{m}^{-}$. These basis vectors are very easily expressible in terms of the one variable differential operator model, where they are sums of two monomials. The basis used by Quesne corresponds to the $V^{-}$ subspaces. Thus our models can be used to carry out the spectral analysis for this PDM system, and they yield a simplification.

\subsection{Classical models for S3}

Now we describe how the methods of classical mechanics lead directly to the quantum models. The classical system S3 on the 2-sphere is determined by the Hamiltonian

$$
\mathcal{H}=\mathcal{J}_{1}^{2}+\mathcal{J}_{2}^{2}+\mathcal{J}_{3}^{2}+\frac{\alpha\left(s_{1}^{2}+s_{2}^{2}+s_{3}^{2}\right)}{s_{3}^{2}},
$$

where $\mathcal{J}_{1}=s_{2} p_{3}-s_{3} p_{2}$ and $\mathcal{J}_{2}, \mathcal{J}_{3}$ are cyclic permutations of this expression. For computational convenience we have imbedded the 2-sphere in Euclidean 3-space. Thus we use the Poisson bracket

$$
\{\mathcal{F}, \mathcal{G}\}=\sum_{i=1}^{3}\left(-\partial_{s_{i}} \mathcal{F} \partial_{p_{i}} \mathcal{G}+\partial_{p_{i}} \mathcal{F} \partial_{s_{i}} \mathcal{G}\right)
$$

for our computations, but at the end we restrict to the sphere $s_{1}^{2}+s_{2}^{2}+s_{3}^{2}=1$. The classical basis for the constants of the motion is

$$
\mathcal{L}_{1}=\mathcal{J}_{1}^{2}+\alpha \frac{s_{2}^{2}}{s_{3}^{2}}, \quad \mathcal{L}_{2}=\mathcal{J}_{1} \mathcal{J}_{2}-\alpha \frac{s_{1} s_{2}}{s_{3}^{2}}, \quad \mathcal{X}=\mathcal{J}_{3}
$$

The structure relations are

$$
\left\{\mathcal{X}, \mathcal{L}_{1}\right\}=-2 \mathcal{L}_{2}, \quad\left\{\mathcal{X}, \mathcal{L}_{2}\right\}=2 \mathcal{L}_{1}-\mathcal{H}+\mathcal{X}^{2}+\alpha, \quad\left\{\mathcal{L}_{1}, \mathcal{L}_{2}\right\}=-2\left(\mathcal{L}_{1}+\alpha\right) \mathcal{X}
$$

and the Casimir relation is

$$
\mathcal{L}_{1}^{2}+\mathcal{L}_{2}^{2}-\mathcal{L}_{1} \mathcal{H}+\mathcal{L}_{1} \mathcal{X}^{2}+\alpha \mathcal{X}^{2}+\alpha \mathcal{L}_{1}=0
$$

From the results of [46] we know that additive separation of variables in the Hamilton-Jacobi equation $\mathcal{H}=E$ is possible in subgroup type coordinates in which $\mathcal{X}, \mathcal{L}_{1}$ or $\mathcal{S}=2\left(\mathcal{L}_{1}-i \mathcal{L}_{2}\right)-$ $\mathcal{H}+\mathcal{X}^{2}$, respectively, are constants of separation. This corresponds to two choices of spherical coordinates and one of horospherical coordinates, respectively. We seek two variable models for the Poisson bracket relations (16), (17). There is also separation in ellipsoidal coordinates (i.e., non-subgroup type coordinates) but we will not make use of this here.

The justification for these models comes from Hamilton-Jacobi theory. The phase space for our problem is 4 -dimensional. Thus it is possible to find canonical variables $\mathcal{H}, \mathcal{I}, \mathcal{Q}, \mathcal{P}$ such 
that $\{\mathcal{I}, \mathcal{H}\}=\{\mathcal{P}, \mathcal{Q}\}=1$ and all other Poisson brackets vanish. In terms of $\mathcal{H}$ and the other canonical variables the Poisson bracket can be expressed as

$$
\{\mathcal{F}, \mathcal{G}\}=-\partial_{\mathcal{H}} \mathcal{F} \partial_{\mathcal{I}} \mathcal{G}+\partial_{\mathcal{I}} \mathcal{F} \partial_{\mathcal{H}} \mathcal{G}-\partial_{\mathcal{Q}} \mathcal{F} \partial_{\mathcal{P}} \mathcal{G}+\partial_{\mathcal{P}} \mathcal{F} \partial_{\mathcal{Q}} \mathcal{G}
$$

(As follows from standard theory [47] one can construct a set of such canonical variables from a complete integral of the Hamilton-Jacobi equation. Our 2D second order superintegrable systems are always multiseparable, and each separable solution of the Hamilton-Jacobi equation provides a complete integral. Thus we can find these canonical variables in several distinct ways.) Now we restrict our attention to the algebra of constants of the motion. This algebra is generated by $\mathcal{H}, \mathcal{L}_{1}, \mathcal{L}_{1}, \mathcal{X}$, subject to the relation (17). Thus, considered as functions of the canonical variables, the constants of the motion are independent of $\mathcal{H}$. If we further restrict the system to the constant energy space $\mathcal{H}=E$ then we can consider $\mathcal{H}$ as non varying and every constant of the motion $\mathcal{F}$ can be expressed in the form $\mathcal{F}(E, \mathcal{Q}, \mathcal{P})$. This means that the Poisson bracket of two constants of the motion, $\mathcal{F}, \mathcal{G}$ can be computed as

$$
\{\mathcal{F}, \mathcal{G}\}=-\partial_{\mathcal{Q}} \mathcal{F} \partial_{\mathcal{P}} \mathcal{G}+\partial_{\mathcal{P}} \mathcal{F} \partial_{\mathcal{Q}} \mathcal{G}
$$

Thus all functions depend on only two canonically conjugate variables $\mathcal{Q}, \mathcal{P}$ and the parameter $E$. This shows the existence and the form of two variable models of conjugate variables. However the proof is not constructive and, furthermore, it is not unique. Two obtain constructive results we will use the strategy of setting $\mathcal{Q}$ equal to one of the constants of the motion that corresponds to separation of variables in some coordinate system, and then use (18) for the Poisson bracket and require that relations (16), (17) hold. In order to make clear that we are computing on the constant energy hypersurface expressed in canonical variables we will use a different notation. We will set $\mathcal{Q}_{E}=c, \mathcal{P}_{E}=\beta$ so, $\mathcal{F}(\mathcal{H}, \mathcal{Q}, \mathcal{P})=f(c, \beta), \mathcal{G}(\mathcal{H}, \mathcal{Q}, \mathcal{P})=g(c, \beta)$, and

$$
\{\mathcal{F}, \mathcal{G}\}_{E}=\{f, g\}=-\partial_{c} f \partial_{\beta} g+\partial_{\beta} f \partial_{c} g .
$$

For our first model we require $X \equiv \mathcal{X}_{E}=c$. Substituting this requirement and $\mathcal{H}=E$ into the structure equations we obtain the result

$$
\begin{aligned}
I: \quad L_{1} & =\frac{1}{2}\left(E-c^{2}-\alpha\right)+\frac{1}{2} \sqrt{c^{4}-2 c^{2}(E+\alpha)+(E-\alpha)^{2}} \sin 2 \beta, \\
X & =c, \quad L_{2}=\frac{1}{2} \sqrt{c^{4}-2 c^{2}(E+\alpha)+(E-\alpha)^{2}} \cos 2 \beta .
\end{aligned}
$$

In this model, and in all other classical models, $\beta$ is not uniquely determined: we can replace it by $\beta^{\prime}=\beta+k(c)$ for any function $k(c)$ and the variables $c$ and $\beta^{\prime}$ remain canonically conjugate.

For a second model we require $L_{1} \equiv\left(\mathcal{L}_{1}\right)_{E}=c$ and proceed in a similar fashion. The result is

$$
\begin{aligned}
I I: \quad L_{1} & =c, \quad L_{2}=\sqrt{c(E-c-\alpha)} \sin (2 \sqrt{c+\alpha} \beta), \\
X & =\sqrt{\frac{c(E-c-\alpha)}{c+\alpha}} \cos (2 \sqrt{c+\alpha} \beta) .
\end{aligned}
$$

For the third and last model we need to diagonalize the symmetry $\mathcal{S}=2\left(\mathcal{L}_{1}-i \mathcal{L}_{2}\right)-\mathcal{H}+\mathcal{X}^{2}$ corresponding to separation in horospherical coordinates. For this it is convenient to rewrite the structure equations (16), (17) in terms of the new basis $\mathcal{S}, \mathcal{L}_{1}+i \mathcal{L}_{2}, \mathcal{X}$ :

$$
\begin{aligned}
& \{\mathcal{S}, \mathcal{X},\}=2 i(\mathcal{S}+\alpha), \quad\left\{\mathcal{S}, \mathcal{L}_{1}+i \mathcal{L}_{2}\right\}=-2 i \mathcal{X}\left(\mathcal{S}-2 \mathcal{X}^{2}+2 \mathcal{H}+3 \alpha\right), \\
& \left\{\mathcal{L}_{1}+i \mathcal{L}_{2}, \mathcal{X}\right\}=-i\left(\mathcal{X}^{2}+2\left(\mathcal{L}_{1}+i \mathcal{L}_{2}\right)-\mathcal{H}+\alpha\right)
\end{aligned}
$$

The Casimir relation is

$$
-2 \mathcal{S}\left(\mathcal{L}_{1}+i \mathcal{L}_{2}\right)-\mathcal{S} \mathcal{X}^{2}+\mathcal{X}^{4}+\mathcal{H} \mathcal{S}-2 \mathcal{X}^{2} \mathcal{H}+\mathcal{H}^{2}-\alpha\left(2\left(\mathcal{L}_{1}+i \mathcal{L}_{2}\right)+\mathcal{S}+3 \mathcal{X}^{2}+\mathcal{H}\right)=0
$$


For model III we set $S=c$ and obtain

$$
\begin{array}{ll}
I I I: & S=c, \quad X=-2 i(c+\alpha) \beta \\
& L_{1}+i L_{2}=8(c+\alpha)^{3} \beta^{4}+2(c+\alpha)(3 \alpha+c+2 E) \beta^{2}-\frac{(c+E)(\alpha-E)}{2(c+\alpha)} .
\end{array}
$$

\subsection{Classical model $\rightarrow$ quantum model}

What have we achieved with these classical models? For one thing they show us how to parameterize the constants of the motion and exhibit their functional dependence. More important for our purposes, they give us a rational means to derive the possible one-variable quantum models. This may seem surprising. How can classical mechanics determine quantum mechanics uniquely? How can structures such as the Wilson family of orthogonal polynomials, containing the Hahn polynomials, be derived directly from classical mechanics? The point is that the structures we are studying are second order superintegrable systems in 2D. In papers [30, 44, 27] it has been shown that there is a 1-1 relationship between the quantum and classical versions for such systems, for all 2D Riemannian spaces. (Similarly there is a 1-1 relationship in 3D for nondegenerate potentials on conformally flat spaces.) The structures are not identical, since as we can see from the examples in this paper, the structure relations in the classical and quantum cases are not identical; there are quantum modifications of the classical equations. Although we know of no direct prescription for their determination, nonetheless the quantum structure equations are uniquely determined by the classical structure equations. Given a second system of second order constants of the motion we write down the corresponding quantum system via the usual correspondence, where products of classical functions are replaced by symmetrized quantum operators, and generate the quadratic algebra by taking repeated commutators. Even order classical symmetries correspond to formally self-adjoint quantum symmetries, and odd order classical symmetries correspond to formally skew-adjoint quantum symmetries. (This relationship no longer holds for third order superintegrable systems [21,40].) We will demonstrate here how to get quantum models from the classical ones that we have derived.

The basic prescription for the transition from the classical case to the operator case is to replace a pair of canonically conjugate variables $c, \beta$ by $c \rightarrow t, \beta \rightarrow \partial_{t}$. (There is no obstruction to quantization for second order superintegrable systems.) Once an appropriate choice of $\beta$ is made in a classical model, we can use this prescription to go to a differential operator model of the quantum structure equations. In particular model III above suggests a operator model such that $S$ is multiplication by $c, X$ is a first order differential operator in $c$ and $L_{1}+i L_{2}$ is a fourth order differential operator. The result, whose existence is implied by the 1-1 classical/quantum relationship for second order superintegrable systems, is

$$
\begin{aligned}
& I I I: \quad S=t, \quad X=-2 i(t+\alpha) \partial_{t}+2 i, \\
& L_{1}+i L_{2}= 8(t+\alpha)^{3} \partial_{t}^{4}+2(t+\alpha)(3 \alpha+t+2 E+9) \partial_{t}^{2}-2(t+5 \alpha+4 E+18) \partial_{t} \\
&+\left(2+\frac{E}{2}-\frac{\alpha}{2}\right)+\frac{E^{2}+2 E(9-\alpha)+(\alpha+12)(\alpha+6)}{2(t+\alpha)} .
\end{aligned}
$$

The leading order differential operators terms agree with the classical case but there are lower order correction terms needed to correct for the noncommutivity of $t$ and $\partial_{t}$. We can realize various irreducible representations of the quadratic algebra by choosing subspaces of functions of $t$ on which the operators act. This model agrees with (10), (11), (12) in the case where $C(n-1, n)=1$ and $C(n+1, n)$ is fourth order. However, there we had a space spanned by a countable number of eigenvectors of the skew-adjoint symmetry $X$ whereas here we want the spectral decomposition of the self-adjoint symmetry $S$ to govern the model. This forces $L_{2}$ to be skew-adjoint and $X$ to be self-adjoint. Thus, though the differential operators are formally 
the same, the Hilbert spaces and the spectral analysis are different. All the representations are infinite-dimensional. One class can be realized by closing the dense subspace of $C^{\infty}$ functions with compact support on $0<t<\infty$ where the measure is $d t / t$. The the spectrum of $S$ is continuous and runs over the positive real axis. Here $X$ also has continuous real spectra covering the full real axis. In particular the generalized eigenfunction of $X$ with real eigenvalue $\lambda$ is proportional to $t^{-i \lambda}$, and $\mu$ is pure imaginary. Thus the spectral analysis of $X$ is given by the Mellin transform. There is a similar irreducible representation defined on $-\infty<t<0$. By a canonical transformation we can also get models of these representations in which both $C(n-1, n)=1$ and $C(n+1, n)$ are second order. (We shall illustrate this explicitly for model I.) Then the spectral decomposition of $S$ is given by the Hankel transform. Since these particular eigenspaces of $H$ admit no discrete spectrum for any of the symmetries of interest, we shall not analyze them further.

Now we consider model I, (19). Due to the presence of trigonometric terms in $\beta$ we cannot realize this as a finite order differential operator model. However, we can perform a hodograph transformation, i.e. use the prescription $\beta \rightarrow t, c \rightarrow-\partial_{t}$ to realize the model. This would seem to make no sense due to the appearance of functions of $c$ under the square root sign. However, before using the prescription we can make use of the freedom to make a replacement $\beta^{\prime}=\beta+g(c)$ which preserves canonical variables. We choose

$$
e^{-2 i \beta} \rightarrow e^{-2 i \beta} / \sqrt{c^{4}-2 c^{2}(E+\alpha)+(E-\alpha)^{2}}
$$

but leave $c$ unchanged. Then we find

$$
\begin{aligned}
L_{1} & =\frac{1}{2}\left(E-c^{2}-\alpha\right)-\frac{i}{4}\left[\left(c^{4}-2 c^{2}(E+\alpha)+(E-\alpha)^{2}\right) e^{2 i \beta}-1\right], \\
L_{2} & =-\frac{i}{4}\left[\left(c^{4}-2 c^{2}(E+\alpha)+(E-\alpha)^{2}\right) e^{2 i \beta}+1\right],
\end{aligned}
$$

with $X$ as before. Now we apply the quantization prescription $\beta \rightarrow t, c \rightarrow-\partial_{t}$ and obtain a model in which both $L_{1}$ and $L_{2}$ are fourth order and $X$ is a first order differential operator. This is, in fact, identical to within a coordinate change to model (21). One might also try to obtain a difference operator model from (22) with the replacement $c \rightarrow t, \beta \rightarrow \partial_{t}$, so that $e^{2 i \beta}$ would become a difference operator. However, this difference operator quantum model is equivalent to what would get from the $\beta \rightarrow t, c \rightarrow-\partial_{t}$ model by taking a Fourier transform. Thus we don't regard it as new.

There is an alternate way to obtain a quantum realization from model I. We use the fact that

$$
c^{4}-2 c^{2}(E+\alpha)+(E-\alpha)^{2}=\left(c^{2}-(E+\alpha)\right)^{2}-4 \alpha
$$

and set

$$
\phi=\arctan \left(\frac{\sqrt{-4 \alpha}}{c^{2}-(E+\alpha)^{2}}\right) .
$$

Now we let $2 \beta \rightarrow 2 \beta+\phi$ to obtain

$$
\begin{aligned}
& L_{1}=\frac{1}{2}\left(E-c^{2}-\alpha\right)+\frac{1}{2}\left(\left(c^{2}-(E+\alpha)^{2}\right) \sin 2 \beta+2 i \sqrt{\alpha} \cos 2 \beta\right), \\
& L_{2}=\frac{1}{2}\left(\left(c^{2}-(E+\alpha)^{2}\right) \cos 2 \beta-2 i \sqrt{\alpha} \sin 2 \beta\right), \quad X=c .
\end{aligned}
$$

Now the prescription $\beta \rightarrow t, c \rightarrow-\partial_{t}$ leads to a quantum realization of $L_{1}, L_{2}$ by second order differential operators. Indeed

$$
L_{1}=\frac{1}{2}(\cos (2 t)-1) \partial_{t}^{2}-8 i \xi \sin (2 t) \partial_{t}+\left(-\frac{E}{2}+64 \xi^{2}+8 i \xi-\frac{1}{4}-\frac{\alpha}{2}\right) \cos (2 t)+\frac{E-\alpha}{2},
$$




$$
\begin{aligned}
& L_{2}=\frac{1}{2} \sin (2 t) \partial_{t}^{2}+8 i \xi \cos (2 t) \partial_{t}+\left(-\frac{E}{2}+64 \xi^{2}+8 i \xi-\frac{1}{4}-\frac{\alpha}{2}\right) \cos (2 t)+\frac{E-\alpha}{2}, \\
& X=\partial_{t} .
\end{aligned}
$$

Here $\xi$ is arbitrary and can be removed via a gauge transformation. The change of variable $\tau=e^{2 i t}$ reduces this model to the form (13). This shows that the flexibility we had in constructing differential operator models from the abstract representation theory by renormalizing our basis vectors $f_{n}$ is replaced in the classical model case by appropriate canonical transformations $c \rightarrow c, \beta \rightarrow \beta+g(c)$. In either case there is essentially only one differential operator model that can be transformed in various ways.

It is clear that model II cannot produce finite order differential operator realizations of the quantum quadratic algebra, due to the intertwining of square root dependence for $c$ and exponential dependence for $\beta$. However, it will produce a difference operator realization via Taylor's theorem: $e^{a \partial_{t}} f(t)=f(t+a)$. To show this explicitly we make a coordinate change such that $2 \sqrt{c+\alpha} \partial_{c}=\partial_{\mathcal{C}}$ in $(20)$, which suggests realizations of the quantum operators in the form

$$
\begin{aligned}
& L_{1} f(t)=\left(t^{2}-\alpha\right) f(t), \quad X f(t)=h(t) f(t+i)+m(t) f(t-i), \\
& L_{2} f(t)=-\frac{i}{2}(i+2 t) h(t) f(t+i)+\frac{i}{2}(-i+2 t) m(t) f(t-i) .
\end{aligned}
$$

A straightforward computation shows that the quantum algebra structure equations are satisfied if and only if

$$
h(t) m(t+i)=\frac{1}{4} \frac{\left(\alpha-t^{2}-i t\right)\left(t^{2}+i t-E\right)}{t(t+i)} .
$$

Since $\alpha=-a^{2}+\frac{1}{4}$ and $E=-(\mu-1+a)^{2}+\frac{1}{4}$ for bounded below representations, we can factor (24) simply to obtain

$$
\begin{aligned}
h(t) m(t+i)= & -\frac{1}{4 t(t+i)}\left(t+\frac{i}{2}+i a\right)\left(t+\frac{i}{2}-i a\right) \\
& \times\left(t+\frac{i}{2}+i \mu+i a\right)\left(t+\frac{3 i}{2}-i \mu-i a\right) .
\end{aligned}
$$

Note that only the product (24) is determined, not the individual factors. Thus we can choose $h(t)$, say, as an arbitrary nonzero function and then determine $m(t)$ from (24). All these modifications of the factors are accomplished by gauge transformations on the representation space: $\tilde{f}(t)=\rho(t) f(t)$ where $\rho(t)$ is the gauge function. If we choose the factors in the form

$$
h(t)=i \frac{\left(\frac{1}{2}-a-i t\right)\left(\mu+a-\frac{1}{2}-i t\right)}{2 t}, \quad m(t)=-i \frac{\left(\frac{1}{2}-a+i t\right)\left(\mu+a-\frac{1}{2}+i t\right)}{2 t},
$$

then we we get exactly the model (15). The finite dimensional model is related by the simple change of variables $t \rightarrow i(t-a+1 / 2), \mu=-m$. In any case, there is only a single solution of these equations, up to a gauge transformation.

\subsection{The classical model for S9}

This is the system on the complex sphere, with nondegenerate potential

$$
V=\frac{a_{1}}{s_{1}^{2}}+\frac{a_{2}}{s_{2}^{2}}+\frac{a_{3}}{s_{3}^{2}}
$$


where $s_{1}^{2}+s_{2}^{2}+s_{3}^{2}=1$. The classical S9 system has a basis of symmetries

$$
\mathcal{L}_{1}=\mathcal{J}_{1}^{2}+a_{2} \frac{s_{3}^{2}}{s_{2}^{2}}+a_{3} \frac{s_{2}^{2}}{s_{3}^{2}}, \quad \mathcal{L}_{2}=\mathcal{J}_{2}^{2}+a_{3} \frac{s_{1}^{2}}{s_{3}^{2}}+a_{1} \frac{s_{3}^{2}}{s_{1}^{2}}, \quad \mathcal{L}_{3}=\mathcal{J}_{3}^{2}+a_{1} \frac{s_{2}^{2}}{s_{1}^{2}}+a_{2} \frac{s_{1}^{2}}{s_{2}^{2}}
$$

where $\mathcal{H}=\mathcal{L}_{1}+\mathcal{L}_{2}+\mathcal{L}_{3}+a_{1}+a_{2}+a_{3}$ and the $\mathcal{J}_{i}$ are defined by $\mathcal{J}_{3}=s_{1} p_{s_{2}}-s_{2} p_{s_{1}}$ and cyclic permutation of indices. The classical structure relations are

$$
\begin{aligned}
\left\{\mathcal{L}_{1}, \mathcal{R}\right\}= & 8 \mathcal{L}_{1}\left(\mathcal{H}+a_{1}+a_{2}+a_{3}\right)-8 \mathcal{L}_{1}^{2}-16 \mathcal{L}_{1} \mathcal{L}_{2}-16 a_{2} \mathcal{L}_{2} \\
& +16 a_{3}\left(\mathcal{H}+a_{1}+a_{2}+a_{3}-\mathcal{L}_{1}-\mathcal{L}_{2}\right) \\
\left\{\mathcal{L}_{2}, \mathcal{R}\right\}= & -8 \mathcal{L}_{2}\left(\mathcal{H}+a_{1}+a_{2}+a_{3}\right)+8 \mathcal{L}_{2}^{2}+16 \mathcal{L}_{1} \mathcal{L}_{2}+16 a_{1} \mathcal{L}_{1} \\
& -16 a_{3}\left(\mathcal{H}+a_{1}+a_{2}+a_{3}-\mathcal{L}_{1}-\mathcal{L}_{2}\right),
\end{aligned}
$$

with $\left\{\mathcal{L}_{1}, \mathcal{L}_{2}\right\}=\mathcal{R}$ and

$$
\begin{aligned}
\mathcal{R}^{2}- & 16 \mathcal{L}_{1} \mathcal{L}_{2}\left(\mathcal{H}+a_{1}+a_{2}+a_{3}\right)+16 \mathcal{L}_{1}^{2} \mathcal{L}_{2}+16 \mathcal{L}_{1} \mathcal{L}_{2}^{2}+16 a_{1} \mathcal{L}_{1}^{2} \\
& +16 a_{2} \mathcal{L}_{2}^{2}+16 a_{3}\left(\mathcal{H}+a_{1}+a_{2}+a_{3}\right)^{2}-32 a_{3}\left(\mathcal{H}+a_{1}+a_{2}+a_{3}\right)\left(\mathcal{L}_{1}+\mathcal{L}_{2}\right) \\
& +16 a_{3} \mathcal{L}_{1}^{2}+32 a_{3} \mathcal{L}_{1} \mathcal{L}_{2}+16 a_{3} \mathcal{L}_{2}^{2}-64 a_{1} a_{2} a_{3}=0
\end{aligned}
$$

Taking $\mathcal{L}_{1}=c, \mathcal{H}=E$ with $c, \beta$ as conjugate variables, we find the model

$$
\begin{aligned}
& \mathcal{L}_{2}=\frac{1}{2}\left(a_{1}+2 a_{2}+E-c\right)-\frac{\left(a_{2}-a_{3}\right)\left(a_{1}+2 a_{2}+2 a_{3}+E\right)}{2\left(c+a_{2}+a_{3}\right)} \\
& +\frac{\sqrt{\left(4 a_{1} a_{2}+4 a_{1} a_{3}+2 c\left(E+a_{1}+a_{2}+a_{3}\right)+4 c a_{1}-\left(E+a_{1}+a_{2}+a_{3}\right)^{2}-c^{2}\right)\left(4 a_{2} a_{3}-c^{2}\right)}}{2\left(a_{2}+a_{3}+c\right)} \\
& \times \cos \left(4 \beta \sqrt{a_{2}+a_{3}+c}\right) .
\end{aligned}
$$

This suggests a difference operator realization of the quantum model.

In the quantum case the symmetry operators $L_{1}, L_{2}, L_{3}$ are obtained from the corresponding classical constants of the motion (26) through the replacements $\mathcal{J}_{k} \rightarrow J_{k}$ where the angular momentum operators $J_{k}$ are defined by $J_{3}=x_{1} \partial_{x_{2}}-x_{2} \partial_{x_{1}}$ and cyclic permutation of indices. Here $H=L_{1}+L_{2}+L_{3}+a_{1}+a_{2}+a_{3}$. The quantum structure relations can be put in the symmetric form

$$
\begin{aligned}
{\left[L_{i}, R\right] } & =4\left\{L_{i}, L_{k}\right\}-4\left\{L_{i}, L_{j}\right\}-\left(8+16 a_{j}\right) L_{j}+\left(8+16 a_{k}\right) L_{k}+8\left(a_{j}-a_{k}\right), \\
R^{2}= & \frac{8}{6}\left\{L_{1}, L_{2}, L_{3}\right\}+-\left(16 a_{1}+12\right) L_{1}^{2}-\left(16 a_{2}+12\right) L_{2}^{2}-\left(16 a_{3}+12\right) L_{3}^{2} \\
& +\frac{52}{3}\left(\left\{L_{1}, L_{2}\right\}+\left\{L_{2}, L_{3}\right\}+\left\{L_{3}, L_{1}\right\}\right)+\frac{1}{3}\left(16+176 a_{1}\right) L_{1} \\
& +\frac{1}{3}\left(16+176 a_{2}\right) L_{2}+\frac{1}{3}\left(16+176 a_{3}\right) L_{3}+\frac{32}{3}\left(a_{1}+a_{2}+a_{3}\right) \\
& +48\left(a_{1} a_{2}+a_{2} a_{3}+a_{3} a_{1}\right)+64 a_{1} a_{2} a_{3} .
\end{aligned}
$$

Here $i, j, k$ are chosen such that $\epsilon_{i j k}=1$ where $\epsilon$ is the pure skew-symmetric tensor, $R=\left[L_{1}, L_{2}\right]$ and $\left\{L_{1}, L_{j}\right\}=L_{i} L_{j}+L_{j} L_{i}$ with an analogous definition of $\left\{L_{1}, L_{2}, L_{3}\right\}$ as a sum of 6 terms. In practice we will substitute $L_{3}=H-L_{1}-L_{2}-a_{1}-a_{2}-a_{3}$ into these equations.

Proceeding exactly as in the S3 case (23), (24), (25), we find that the difference operator analogy of (27) for the quantum quadratic algebra is

$$
L_{1}=4 t^{2}-\frac{1}{2}+\beta^{2}+\gamma^{2},
$$




$$
\begin{aligned}
& L_{2}=h(t) T^{i}+m(t) T^{-i}+\ell(t) \\
& =\frac{\left[-4 \alpha^{2}-8 \alpha-4+4 \mathcal{E}^{2}+16 i(\alpha+1) t+16 t^{2}\right](\beta+1+\gamma-2 i t)(\beta-1-\gamma+2 i t)}{1024 t(t+i)(2 t+i)^{2}} \\
& \times\left[-4 \alpha^{2}-4+8 \alpha+4 \mathcal{E}^{2}+16 i(1-\alpha) t+16 t^{2}\right](\beta+1-\gamma-2 i t)(\beta-1+\gamma+2 i t) T^{i}+T^{-i} \\
& +\left[-2 t^{2}-\frac{1}{2} \mathcal{E}^{2}-\frac{1}{2} \beta^{2}+\frac{1}{2} \alpha^{2}+\frac{1}{2} \gamma^{2}+\frac{\left(\gamma^{2}-\beta^{2}\right)\left(-4 \alpha^{2}+4 \mathcal{E}^{2}\right)}{8\left(1+4 t^{2}\right)}\right],
\end{aligned}
$$

where

$$
a_{1}=\frac{1}{4}-\alpha^{2}, \quad a_{2}=\frac{1}{4}-\beta^{2}, \quad a_{3}=\frac{1}{4}-\gamma^{2}, \quad H=\frac{1}{4}-\mathcal{E}^{2} .
$$

The quadratic terms factor into simple linear terms, and just as in the S3 case, it is only $\ell(t)$ and the product $h(t) m(t+i)$ that is uniquely determined. We can change the individual factors $h(t), m(t)$ by a gauge transformation. With the change of variable $t=i \tau$ and a gauge transformation to an operator with maximal symmetry in $\tau$, we obtain the standard model

$$
\begin{aligned}
& h(t)=\tilde{h}(\tau)=\frac{(A+\tau)(B+\tau)(C+\tau)(D+\tau)}{4 \tau(\tau+1 / 2)}, \\
& m(t)=\tilde{m}(\tau)=\frac{(A-\tau)(B-\tau)(C-\tau)(D-\tau)}{4 \tau(\tau-1 / 2)}, \\
& A=\frac{\mathcal{E}+\alpha+1}{2}, \quad B=\frac{\mathcal{E}-\alpha+1}{2}, \quad C=\frac{\beta+\gamma+1}{2}, \quad D=\frac{\beta-\gamma+1}{2} .
\end{aligned}
$$

It follows that $L_{2}=\tilde{h}(\tau) E^{+1}+\tilde{m}(\tau) E^{-1}+\tilde{\ell}(\tau)$ is a linear combination of $L_{1}$ and the difference operator whose eigenfunctions are the Wilson polynomials, just as found in [39]. Here $E^{s} f(\tau)=$ $f(\tau+s)$.

\section{Conclusions and prospects}

This paper consists of two related parts. In the first part we have studied the representation theory for the quadratic algebra associated with a $2 \mathrm{D}$ second order quantum superintegrable system with degenerate potential, namely S3. We have classified the possible finite-dimensional representations and infinite dimensional bounded below representations, i.e., those with a lowest weight vector. Then we have constructed the possible Hilbert space models for these representations, in terms of differential operators or of difference operators acting on spaces of functions of one complex variable. These models make it easy to find raising and lowering operators for the representations and to uncover relationships between the algebras and families of orthogonal polynomials. Here S3 has been treated as an example of a degenerate potential superintegrable system. The example S9 of a nondegenerate potential was treated in [39]. In 2D there are 13 equivalence classes of superintegrable systems with nontrivial potentials: 7 nondegenerate and 6 degenerate. Results for all of these cases will be included in the thesis of the third author.

In the second part of this work we have taken up the study of models of the quadratic algebras associated with the classical second order superintegrable systems. In each model there is only a single pair of canonically conjugate variables, rather than the 2 pairs in the original classical system. We showed, based on classical Hamilton-Jacobi theory, that such models always exist. Then we described a procedure to derive the one variable models for the quantum quadratic algebras from the models for the classical quadratic algebras. Since it is known that there is a 1-1 relationship between classical and quantum second order superintegrable systems (even though the algebras are not the same), it is not too surprising that one should be able to compute the quantum models from the classical models. However, we have made this explicit. 
We applied this procedure not only to obtain the differential and difference operator models for system S3, but also for the generic system S9. For S9 we showed that there is a difference operator model associated with general Wilson polynomials, but no differential operator model. This construction demonstrates that the theory of general Wilson polynomials is imbedded in classical mechanics in a manner quite different from the usual group theory (Racah polynomial) approach.

There is much more work to be done. Once the models are worked out and the corresponding functional Hilbert spaces are constructed, usually Hilbert spaces with kernel function, then one needs to find intertwining operators that map the model space to the space on which the quantum Schrödinger operator is defined. Also,we have demonstrated how to determine the classical models and show how they quantize in a unique fashion. A puzzle here is that we are finding classical models corresponding to non-hypergeometric type variable separation. These classical models typically involve elliptic functions. We do not yet understand how they can be quantized. They clearly do not lead to differential or ordinary difference operator quantum models.

Another part of our effort is to study the structure of quadratic algebras corresponding to 3D nondegenerate superintegrable systems, and to find two variable models for them. This is a much more difficult problem than in 2D, where it led to general Wilson and Racah polynomials, among other models. The quadratic algebra still closes at order 6 but now there are 6 linearly second order symmetries, rather than 3 , and they are functionally dependent, satisfying a polynomial relation of order 8 . There are 4 commutators, instead of 1 . For the models we expect to find multivariable extensions of Wilson polynomials, among many other constructs.

\section{References}

[1] Wojciechowski S., Superintegrability of the Calogero-Moser system, Phys. Lett. A 95 (1983), 279-281.

[2] Evans N.W., Superintegrability in classical mechanics, Phys. Rev. A 41 1990, 5666-5676. Evans N.W., Group theory of the Smorodinsky-Winternitz system, J. Math. Phys. 32 (1991), 3369-3375.

[3] Evans N.W., Super-integrability of the Winternitz system, Phys. Lett. A 147 (1990), 483-486.

[4] Friš J., Mandrosov V., Smorodinsky Ya.A., Uhlír M., Winternitz P., On higher symmetries in quantum mechanics, Phys. Lett. 16 (1965), 354-356.

[5] Bonatos D., Daskaloyannis C., Kokkotas K., Deformed oscillator algebras for two-dimensional quantum superintegrable systems, Phys. Rev. A 50 (1994), 3700-3709, hep-th/9309088.

[6] Daskaloyannis C., Quadratic Poisson algebras of two-dimensional classical superintegrable systems and quadratic associative algebras of quantum superintegrable systems, J. Math. Phys. 42 (2001), 1100-1119, math-ph/0003017.

[7] Letourneau P., Vinet L., Superintegrable systems: polynomial algebras and quasi-exactly solvable Hamiltonians, Ann. Phys. 243 (1995), 144-168.

[8] Rañada M.F., Superintegrable $n=2$ systems, quadratic constants of motion, and potentials of Drach, J. Math. Phys. 38 (1997), 4165-4178.

[9] Kalnins E.G., Miller W. Jr., Williams G.C., Pogosyan G.S., On superintegrable symmetry-breaking potentials in $n$-dimensional Euclidean space, J. Phys. A: Math. Gen. 35 (2002), 4655-4720.

[10] Kalnins E.G., Miller W. Jr., Pogosyan G.S., Completeness of multiseparable superintegrability in $E_{2, C}$, J. Phys. A: Math. Gen. 33 (2000), 4105-4120.

[11] Kalnins E.G., Miller W. Jr., Pogosyan G.S., Completeness of multiseparable superintegrability on the complex 2-sphere, J. Phys. A: Math. Gen. 33 (2000), 6791-6806.

[12] Koenigs G., Sur les géodésiques a intégrales quadratiques, A note appearing in "Lecons sur la théorie générale des surfaces", G. Darboux, Vol. 4, Chelsea Publishing, 1972, 368-404.

[13] Calogero F., Solution to the one-dimensional $N$-body problems with quadratic and/or inversely quadratic pair potentials, J. Math. Phys. 12 (1971), 419-436. 
[14] Rauch-Wojciechowski S., Waksjö C., What an effective criterion of separability says about the Calogero type systems, J. Nonlinear Math. Phys. 12 (2005), suppl. 1, 535-547.

[15] Kalnins E.G., Kress J.M., Miller W. Jr., Pogosyan G.S., Completeness of superintegrability in twodimensional constant curvature spaces, J. Phys. A: Math. Gen. 34 (2001), 4705-4720, math-ph/0102006.

[16] Zhedanov A.S., "Hidden symmetry" of Askey-Wilson polynomials, Theoret. and Math. Phys. 89 (1991), $1146-1157$.

[17] Granovskii Ya.I., Zhedanov A.S., Lutsenko I.M., Quadratic algebras and dynamics in curved spaces. I. Oscillator, Theoret. and Math. Phys. 89 (1992), 474-480.

[18] Granovskii Ya.I., Zhedanov A.S., Lutsenko I.M., Quadratic algebras and dynamics in curved spaces. II. The Kepler problem, Theoret. and Math. Phys. 91 (1992), 604-612.

[19] Quesne C., Generalized deformed parafermions, nonlinear deformations of so(3) and exactly solvable potentials, Phys. Lett. A 193 (1994), 245-250.

[20] Tempesta P., Turbiner A.V., Winternitz P., Exact solvability of superintegrable systems, J. Math. Phys. 42 (2001), 4248-4257.

[21] Gravel S., Winternitz P., Superintegrability with third-order integrals in quantum and classical mechanics, J. Math. Phys. 43 (2002), 5902-5912, math-ph/0206046.

[22] Ballesteros A., Herranz F., Santander M., Sanz-Gil T., Maximal superintegrability on N-dimensional curved spaces, J. Phys. A: Math. Gen. 36 (2003), L93-L99, math-ph/0211012.

[23] Kalnins E.G., Kress J.M., Miller W. Jr., Second order superintegrable systems in conformally flat spaces. I. 2D classical structure theory, J. Math. Phys. 46 (2005), 053509, 28 pages.

[24] Kalnins E.G., Kress J.M., Miller W. Jr., Second order superintegrable systems in conformally flat spaces. II. The classical 2D Stäckel transform, J. Math. Phys. 46 (2005), 053510, 15 pages.

[25] Kalnins E.G., Kress J.M., Miller W. Jr., Second order superintegrable systems in conformally flat spaces. III. 3D classical structure theory, J. Math. Phys. 46 (2005), 103507, 28 pages.

[26] Kalnins E.G., Kress J.M., Miller W. Jr., Second order superintegrable systems in conformally flat spaces. IV. The classical 3D Stäckel transform and 3D classification theory, J. Math. Phys. 47 (2006), 043514, 26 pages.

[27] Kalnins E.G., Kress J.M., Miller W. Jr., Second order superintegrable systems in conformally flat spaces. V. 2D and 3D quantum systems, J. Math. Phys. 47 (2006), 093501, 25 pages.

[28] Kalnins E.G., Miller W. Jr., Pogosyan G.S., Exact and quasi-exact solvability of second order superintegrable systems. I. Euclidean space preliminaries, J. Math. Phys. 47 (2006), 033502, 30 pages, math-ph/0412035.

[29] Kalnins E.G., Kress J.M., Winternitz P., Superintegrability in a two-dimensional space of non-constant curvature, J. Math. Phys. 43 (2002), 970-983, math-ph/0108015.

[30] Kalnins E.G., Kress J.M., Miller W. Jr., Winternitz P., Superintegrable systems in Darboux spaces, J. Math. Phys. 44 (2003), 5811-5848, math-ph/0307039.

[31] Daskaloyannis C., Ypsilantis K., Unified treatment and classification of superintegrable systems with integrals quadratic in momenta on a two dimensional manifold, J. Math. Phys. 47 (2006), 042904, 38 pages, math-ph/0412055.

[32] Horwood J.T., McLenaghan R.G., Smirnov R.G., Invariant classification of orthogonally separable Hamiltonian systems in Euclidean space, Comm. Math. Phys. 259 (2005), 679-709, math-ph/0605023.

[33] Tempesta P., Winternitz P., Miller W., Pogosyan G. (Editors), Superintegrability in classical and quantum systems, CRM Proceedings Lecture Notes, Vol. 37, American Mathematical Society, Providence, RI, 2004.

[34] Kalnins E.G., Kress J.M., Miller W. Jr., Nondegenerate 2D complex Euclidean superintegrable systems and algebraic varieties, J. Phys. A: Math. Theor. 40 (2007), 3399-3411.

[35] Kalnins E.G., Kress J.M., Miller W. Jr., Fine structure for 3D second order superintegrable systems: 3parameter potentials, J. Phys. A: Math. Theor. 40 (2007), 5875-5892.

[36] Kalnins E.G., Kress J.M., Miller W. Jr., Nondegenerate 3D complex Euclidean superintegrable systems and algebraic varieties, J. Math. Phys. 48 (2007), 113518, 26 pages, arXiv:0708.3044.

[37] Quesne C., Quadratic algebra approach to an exactly solvable position-dependent mass Schrödinger equation in two dimensions, SIGMA 3 (2007), 067, 14 pages, arXiv:0705.2577.

[38] Daskaloyannis C., Tanoudis Y., Quantum superintegrable systems with quadratic integrals on a two dimensional manifold, J. Math. Phys. 48 (2007), 072108, 22 pages, math-ph/0607058. 
[39] Kalnins E.G., Miller W. Jr., Post S., Wilson polynomials and the generic superintegrable system on the 2-sphere, J. Phys. A: Math. Theor. 40 (2007), 11525-11538.

[40] Gravel S., Hamilton separable in Cartesian coordinates and third-order integrals of motion, J. Math. Phys. 45 (2004), 1003-1019.

[41] Ballesteros A., Herranz F.J., Universal integrals for superintegrable systems on $N$-dimensional spaces of constant curvature, J. Phys. A: Math. Theor. 40 (2007), F51-F59, math-ph/0610040.

[42] Fordy A.P., Quantum super-integrable systems as exactly solvable models, SIGMA 3 (2007), 025, 10 pages, math-ph/0702048.

[43] Kalnins E.G., Miller W. Jr., Pogosyan G.S., Exact and quasi-exact solvability of second order superintegrable quantum systems. II. Connection with separation of variables, J. Math. Phys. 48 (2007), 023503, 20 pages.

[44] Kress J.M., Equivalence of superintegrable systems in two dimensions, Phys. Atomic Nuclei 70 (2007), $560-566$.

[45] Andrews G.E., Askey R., Roy R., Special functions, Encyclopedia of Mathematics and Its Applications, Cambridge University Press, Cambridge, UK, 1999.

[46] Kalnins E.G., Separation of variables for Riemannian spaces of constant curvature, Pitman, Monographs and Surveys in Pure and Applied Mathematics, Vol. 28, Longman, Essex, England, 1986.

[47] Arnold V.I., Mathematical methods of classical mechanics (translated by K. Vogtmann and A. Weinstein), Graduate Texts in Mathematics, Vol. 60, Springer-Verlag, New York, 1978. 